\title{
Introduction to the Special Issue on Micro- and Nanofabrication Techniques
}

Welcome to this special issue of the Annals of Biomedical Engineering with a focus on applying the techniques of nano- and microfabrication to living cells. The techniques of nano- and microtechnology offer many new possibilities to probe issues that should reveal a more in-depth understanding of biological systems and function. Here we provide examples of application of these techniques to living cells and cellular components. In many cases, this deeper understanding of biological systems will result in new technologies that will benefit society in general.

The primary advantages of nano- and microsystems for biological studies include the ability to:

(1) control multiple inputs to a biological system in space and time;

(2) probe a system at a length scale appropriate to the biological system (e.g., $10-100 \mathrm{~nm}$ for molecules; $10 \mu \mathrm{m}$ for a living cell, and 10 $200 \mu \mathrm{m}$ for a multicellular construct);

(3) facilitate higher throughput assays using smaller amounts of valuable cells, tissue constructs, or biological molecules and thus increase the data necessary to support a robust understanding of the biology; and

(4) create devices with integrated sensors to measure biological responses, particularly highly localized responses.

The papers in this issue focus on model "organs", particularly for probing therapeutics and toxicology, understanding and controlling stem cell biology, developing appropriate diagnostics and sensors, and use of nanoparticles. A common theme in many of these papers is the use of microfluidics to introduce stimuli to cells in a predetermined manner. Microfluidics has become an increasingly critical component of working with biological systems at the microscale.

Examples include the work by Takayama and colleagues reviewing efforts to extend tissue engineering platforms to simulating organs and their function. Leclerc and Prot focus on the development of a novel approach to microfabricate effective "liver" chips. Goral and Yuen review prior efforts for microfabricated mimics of the liver and its function. Hickman and colleagues discuss the design of a microfabricated system for a functional model of the lung. Jean and colleagues describe a system to mimic an injured central nervous system neuron and to test strategies for regeneration. Greenman and colleagues explore the development of a microfluidic system for testing the response of head and neck squamous "tumors" to various drugs and as a potential pre-clinical model for creating personalized treatment regimens. Sung and Shuler review efforts to use the techniques of microfabrication to not only build mimics of specific organs and tissues, but also to build multi organ models that begin to mimic whole body response to various drugs or drug mixtures. Khadenhosseni and colleagues describe approaches to control the stem cell

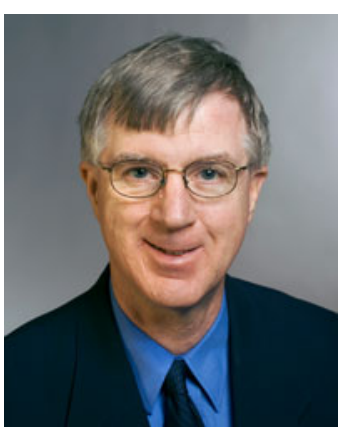

Michael L. Shuler.

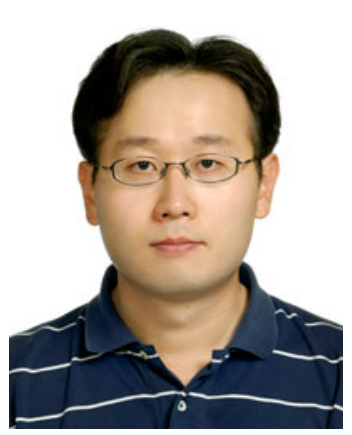

Jong Hwan Sung. environment and hence direct stem cell biology towards a goal. Kim and $\mathrm{Wu}$ discuss microfluidic devices for measuring chemotaxis using one axis based on establishing stable chemical gradients and a second to quantify cellular responses to the gradients. Jin and colleagues study inflammatory responses to sepsis using MRI and a novel superparamagnetic iron oxide-based nanoparticle that localizes to specific cell surface molecules. Suh and colleagues report on a novel approach to micropattering of various polymers and tunable elastic moduli on textured surfaces to provide an increasingly physiologically relevant microenvironment for living cells. Kelly and Craighead review the application of sophisticated nanofabrication approaches to study the response of plasma membranes to topological cues, particularly in terms of signal processing. Lee and colleagues review the use of microfluidic MEMS technology for sample preparation, nucleic acid amplification and detection and rapid diagnosis applied to DNA and RNA. Park and colleagues conclude the issue with an 
overview of progress on the development of biosensors for biomedical applications.

The future of nano- and microscale systems will be laid on many of the ideas in this issue. Indeed DARPA and NIH have recently had calls for essentially a "Human-on-a-Chip" type technology for improved methodology for drug development and evaluation. Many other applications related to personalized medicine are likely outcomes of this type of research. Rapid, simple, cheap diagnostics are also another product that is all but assured. We believe that the marriage of nano- and microtechnology with biological systems will lay the basis for significant improvements in human health.

Michael L. Shuler

Cornell University

Electronic mail: mls50@cornell.edu

Jong Hwan Sung

Chemical Engineering

Hongik University 